\title{
Does the physical disector method provide an accurate estimation of sensory neuron number in rat dorsal root ganglia?
}

\author{
Sibylle Delaloye ${ }^{\mathrm{b}, 1}$, Rudolf Kraftsik ${ }^{\mathrm{a}, 1}$, Thierry Kuntzer ${ }^{\mathrm{b}}$, Ibtissam Barakat-Walter ${ }^{\mathrm{a}, \mathrm{b}, *}$ \\ a Department of Cell Biology and Morphology, University of Lausanne, 1005, Lausanne, Switzerland \\ ${ }^{\mathrm{b}}$ Laboratory of Neurology Research, University Hospital of Lausanne, 1011 Lausanne, Switzerland
}

\section{A R T I C L E I N F O}

\section{Article history:}

Received 7 July 2008

Received in revised form 28 August 2008

Accepted 1 September 2008

\section{Keywords:}

Stereological methods

Direct counting method

Three-dimensional reconstruction

Calibration of physical dissector method

Computer ganglion model

Total number of sensory neurons

Dorsal root ganglia

\begin{abstract}
A B S T R A C T
The physical disector is a method of choice for estimating unbiased neuron numbers; nevertheless, calibration is needed to evaluate each counting method. The validity of this method can be assessed by comparing the estimated cell number with the true number determined by a direct counting method in serial sections. We reconstructed a $1 / 5$ of rat lumbar dorsal root ganglia taken from two experimental conditions. From each ganglion, images of 200 adjacent semi-thin sections were used to reconstruct a volumetric dataset (stack of voxels). On these stacks the number of sensory neurons was estimated and counted respectively by physical disector and direct counting methods. Also, using the coordinates of nuclei from the direct counting, we simulate, by a Matlab program, disector pairs separated by increasing distances in a ganglion model. The comparison between the results of these approaches clearly demonstrates that the physical disector method provides a valid and reliable estimate of the number of sensory neurons only when the distance between the consecutive disector pairs is $60 \mu \mathrm{m}$ or smaller. In these conditions the size of error between the results of physical disector and direct counting does not exceed $6 \%$. In contrast when the distance between two pairs is larger than $60 \mu \mathrm{m}(70-200 \mu \mathrm{m})$ the size of error increases rapidly to $27 \%$.

We conclude that the physical dissector method provides a reliable estimate of the number of rat sensory neurons only when the separating distance between the consecutive dissector pairs is no larger than $60 \mu \mathrm{m}$.
\end{abstract}

(C) 2008 Elsevier B.V. All rights reserved.

\section{Introduction}

Because the total neuron number is an accepted parameter for evaluating neuronal survival or neuronal loss, several methods have been devised for determining the number of surviving neurons from histological sections (Tessler et al., 1985; Arvidsson et al., 1986; Liss et al., 1994; Ljungberg et al., 1999; Pu et al., 1999; McKay et al., 2002; Schmitz and Hof, 2005). Previously the main methods to estimate neuron number were based on two-dimensional microscopic images and involved assumptions about particle shape and distribution. The most commonly used method involved determining nucleolar number followed by the application of a correction factor to convert such counts to neuronal number (Abercrombie, 1946; Konigsmark, 1970). However, the shortcoming of these meth-

* Corresponding author at: Laboratoire de recherche neurologique, service de neurology, CHUV, Rue du Bugnon 46, 1011 Lausanne, Switzerland.

Tel.: +41 2131412 78; fax: +41213144138.

E-mail address: Ibtissam.walter@unil.ch (I. Barakat-Walter).

1 SD and RK have contributed equally to this work. ods is that the assumptions are not completely met, and therefore, the methods are biased (de Groot and Bierman, 1986; Mouton, 2002; von Bartheld, 2002).

In 1984, a further counting method, called the physical disector method, was devised (Sterio, 1984), and this stereological method soon became the accepted way of determining the number of neurons. This method is particularly useful when objects to be counted are large relative to section thickness, and when their dimensions are unknown, or highly variable. Moreover, it can be used to count neurons without having to make any assumptions about their size, shape or orientation (West, 1999a, 2002; Guillery and August, 2002; Schmitz and Hof, 2005). This method based on a three-dimensional (3D) stereological probe, uses two sections, hence the name disector. Only the objects that are observable in the first section of the pair, but not observable in the second section are counted. This is a simple method for avoiding double counts, and its particular strength is that it is completely independent of the size and shape of the objects being counted. However, the use of this method requires that the investigator must be able to identify the sectional profiles that belong to the same object, and there must be at least one section in which it is possible to identify each object (West, 1993). This 
method has been widely applied to estimate the number of neurons in the nervous system. We also used the physical disector method to estimate the number of surviving axotomized sensory neurons in lumbar dorsal root ganglia (DRG), following adult rat sciatic nerve transection and local thyroid hormone (3,3',5-triiodo-L-thyronine, $\mathrm{T}_{3}$ ) administration (Schenker et al., 2003).

Although the physical disector method has important theoretical advantages, as summarized by several investigators (Howard and Reed, 1998; West, 1999b; Geuna, 2000), it is not a foolproof solution for every counting task (Saper, 1997). Therefore, each method should be validated by a calibration to reveal and eventually minimize the counting error (Coggeshall et al., 1990; Farel, 2002; von Bartheld, 2002). Obviously this is the first part of the experimental design, preceding the choice of the sample size for the experimental groups. The most precise method of validation is to compare the results of the method to be calibrated with the complete reconstruction of the organ (structure) of interest. The complete serial reconstruction gives the true number of neurons because there is no sampling or estimating (Pover and Coggeshall, 1991). Of course this calibration is rarely done because complete serial reconstruction requires intensive labour even using the modern powerful desktop computers and usually cannot be completed in a reasonable timeframe. What the investigator can do is to reconstruct a part of the organ or tissue and compare this to the results of other counting methods. With such an analysis it will be able to recognize, and eventually eliminate, most sources of bias.

In the present work, to test the accuracy of the physical disector method we compared the results of physical disector to those obtained by using direct counts derived from a voxel reconstruction of a part of a DRG. A stack of 200 adjacent sections from PBS-control and from $\mathrm{T}_{3}$-treated ganglia was used to reconstruct a volumetric dataset. On these stacks we estimated and counted the number of sensory neurons by physical disector and direct counting methods respectively. In addition, we devised a computer model of a ganglion in order to simulate the influence of separating distances between the two consecutive disector pairs on the estimation of the number of neurons.

\section{Materials and methods}

\subsection{Surgical procedures}

All animal procedures were conducted according to the local guidelines for care and use of experimental animals. To reduce the number of animals used and to directly compare the results of this study with our previous results we used the same DRG sections described by Schenker et al. (2003). In brief, 10-week-old Wistar rats were anaesthetized. The skin of the right leg was cut followed by a longitudinal cut made through the biceps femoris muscle. The right sciatic nerve was transected near to the vertebral column, and a small piece $(6 \mathrm{~mm})$ of the nerve removed. The proximal and distal stumps of the transected nerve (approximately $1 \mathrm{~mm}$ ) were secured into the ends of $10 \mathrm{~mm}$ of sterile silicone tubes with internal diameter of $1.5 \mathrm{~mm}$ and external diameter of $3 \mathrm{~mm}$ (Silclear, Medical Grade tubing, Midland, MI, U.S.A.). The transected nerve was held in place with three polyamide epineural sutures (8-0 Ethicon) at each end, leaving an $8 \mathrm{~mm}$ long gap between the nerve stumps. After placement, the tubes were filled with either a neutral $\mathrm{pH}$ sterile solution of 3,3',5-triiodo-L-thyronine, Sigma ( $1 \mathrm{mg}$ of $\mathrm{T}_{3}$ was dissolved in $1 \mathrm{ml} 0.01 \mathrm{~N} \mathrm{NaOH}$, then the solution neutralized with $0.01 \mathrm{~N} \mathrm{HCl}$, final $\mathrm{pH}$ is 7.5), or with phosphate-buffered saline (PBS) as a control. In our experiments, the $\mathrm{T}_{3}$-treated rats, and PBS controls were taken from the same litter. The overlying muscle and skin of the thigh were sutured. The animals were allowed to survive for 45 days.

\subsection{Processing for light microscopic and morphometric analysis}

Forty-five days after surgery, rats were deeply anaesthetized, then transcardially perfused with $0.1 \mathrm{M}$ phosphate-buffered saline
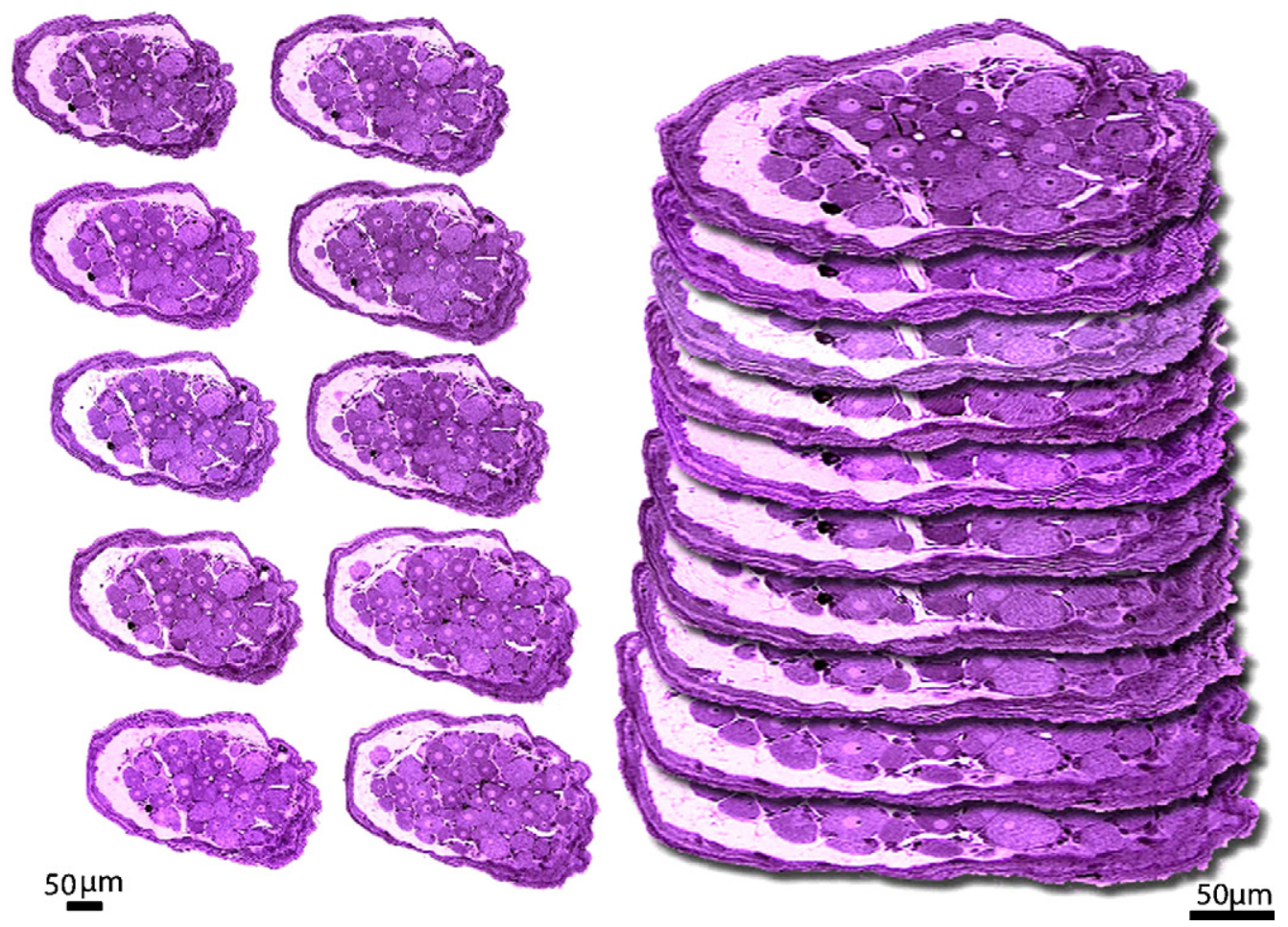

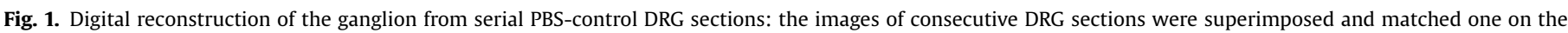
other using neuronal nuclear profiles as well as other landmarks such as the edge of the DRG section, blood vessels etc. 
containing $0.1 \%$ heparin and $0.1 \%$ procaine, followed by a mixture of $3 \%$ paraformaldehyde and $1.5 \%$ glutaraldehyde in $0.1 \mathrm{M}$ PBS at $\mathrm{pH} 7.4$, as described in our previous work (Schenker et al., 2003). The axotomized transected lumbar dorsal root ganglia (L5) were removed from $\mathrm{PBS}$-control and $\mathrm{T}_{3}$-treated rats, then post-fixed for $2 \mathrm{~h}$ at $4{ }^{\circ} \mathrm{C}$ in the respective fixative. After dehydration through increasing ethanol concentrations, the ganglia were oriented in the same direction along their long axis and embedded in Epon. Each entire ganglion was cut into serial longitudinal semi-thin sections of $1 \mu \mathrm{m}$ thickness, comprising between 800 and 1000 sections. Then the sections were stained with $0.1 \%$ Toluidine blue for $10 \mathrm{~s}$ at $80^{\circ} \mathrm{C}$; this staining allows to visualize the nuclei and nucleolar profiles of the neuronal cells. Other ganglia were cut into serial cross semi-thin sections ( $1 \mu \mathrm{m}$ thick) perpendicularly to the long axis of the dorsal root. In this case the number of the sections was 2400.

\subsection{Digital reconstruction of the ganglion from serial images of DRG sections}

About 200 adjacent sections ( $1 \mu \mathrm{m}$ thick) were taken from one PBS-control and one $T_{3}$-treated ganglion starting with the section in which the first neuronal profile was visible. Each histological section was scanned systematically and overlapping pictures of the microscope field were taken with a Sony 930XP video camera, at a magnification of $10 \times$ using the Neurolucida (Microbrightfield Inc., USA) program. The partial images were combined using the Adobe Photoshop (Adobe Systems Inc., USA) program, then these complete images of consecutive sections were best fit one on top of the other, based on their contours and nuclei profiles as well as other landmarks, (blood vessels, nucleoli, etc.) and using the transparency mode of the top layer (the overlay mode in Adobe Photoshop). The procedure was repeated throughout the whole stack to create a three-dimensional reconstruction (a volumetric dataset) of a part $(1 / 5)$ of the ganglion. These aligned 3D stacks were used in the Imaris (Bitplane AG, Zurich, $\mathrm{CH}$ ) program to obtain an interactive visualization of the reconstructed stack, and to control the quality of the $3 \mathrm{D}$ reconstruction.

Two methods were used either to determine or to estimate the total number of neurons in each 3D stack: direct counting and physical disector. To ensure that the counts were unbiased (blind), the observer did not know which treatment had been used for each set of images.

(i) Direct counting method: this method is based on a direct identification and counting of nuclei from the digital images of the 3D image stacks using the confocal module of the Neurolucida program (Microbrightfield Inc.). In the PBS or $\mathrm{T}_{3}$ DRG stacks, the nuclei, with identified nucleoli, were marked once, and the markers remained visible when the nucleus was followed in subsequent sections. New unmarked nuclei with nucleoli in the next sections were then added and followed until all the nuclei with nucleoli were identified. To facilitate the identification and marking of each nucleus found in the stack of 200 sections, each stack was divided into 5 smaller overlapping stacks. The nuclei were first followed and marked in each small stack. All the coordinates of the markers were saved in a file to be used in a computer model. Then, the total number of neurons in the whole PBS or $\mathrm{T}_{3}$ stacks was counted using the NeuroExplorer (Microbrightfield Inc.) program.

(ii) Physical disector method: this method is based on sampling sections, called disector pairs. Twenty consecutive disector pairs were used from each stack. Since on the DRG sections the mean diameter of the nuclei (of large and small neurons) is around $20 \mu \mathrm{m}$, this permitted us to use sections separated by $8 \mu \mathrm{m}$ as physical disector pairs. This design allows estimation of the number of neurons by an unbiased approach. The estimation of the number of neurons was made using a protocol based on Neurolucida software (Microbrightfield Inc.) as described in our previous work (Schenker et al., 2003). To be sure that the random selection of the first section of the first pair gives an equal representation of all DRG regions being sampled, three independent estimations starting by the 3rd, the 5th or the 7th section of each stack were carried out. To investigate the role of the separating distance between the two consecutive pairs, we estimated the number of neurons by physical disector using different distances between the consecutive pairs $(0,10,20,30$, 40 or $50 \mu \mathrm{m})$. We then compared neuron number obtained by physical disector and the true number of neurons determined by direct counting in the same DRG sections.

\subsection{Creating a computer model by using the Matlab program}

From the coordinates of markers recorded on the stack of 200 consecutive longitudinal DRG sections by the direct counting method, we created a computer model for the control and the $\mathrm{T}_{3}$-treated ganglia, using the Matlab (The MathWorks Inc., USA) program. Therefore, our computer model uses the threedimensional coordinates of the markers of nuclei centers. The nuclei were simulated by software as spheres of $20 \mu \mathrm{m}$ diameter, centred on the marker coordinates. The computer ganglion model is included in a rectangular parallelepiped of $200 \mu \mathrm{m}$ depth, $1300 \mu \mathrm{m}$ length and $800 \mu \mathrm{m}$ width. In this ganglion model we simulated about 130 disector pairs of $10 \mu \mathrm{m}$ thickness taken perpendicularly to the long axis of the reconstructed ganglion. We estimated the neuron number by the physical disector method by increasing the separating distance between two consecutive pairs from 0 to $200 \mu \mathrm{m}$ and as a consequence, reducing the number of analysed pairs from 130 to 6 . The results of these simulations were compared to the total number of neurons obtained by direct counting.

\section{Results}

The major question addressed by the present paper is: does the physical disector method, used to estimate neuronal number, yield an accurate estimate when compared with direct counting? For this purpose the number of sensory neurons estimated by the physical disector method for various distances in defined stacks of DRG sections was compared with the true number of neurons determined by the direct counting method in the same DRG stacks.

\subsection{Digital reconstruction of the ganglion from DRG serial sections}

The images of 200 consecutive cross-sections taken either from one PBS-control or one $\mathrm{T}_{3}$-treated ganglion were used to create a $3 \mathrm{D}$ stack reconstruction (set of voxels) for each experimental condition. The images were superimposed one on the other using neuronal nuclear profiles as well as other landmarks such as the edge of the DRG section, blood vessels etc. (Fig. 1). Then the number of neurons was determined in each stack by direct counting and by the physical disector methods.

\subsection{Determination of the true number of sensory neurons by direct counting method}

The analysis of several sections by light microscopy showed that there are no detectable changes in the morphology of neurons between PBS-control (Fig. 1) and $\mathrm{T}_{3}$-treated DRG (Fig. 2). In both conditions sensory neurons can be easily identified and differentiated from non-neuronal cells (Schwann and satellite cells) on 


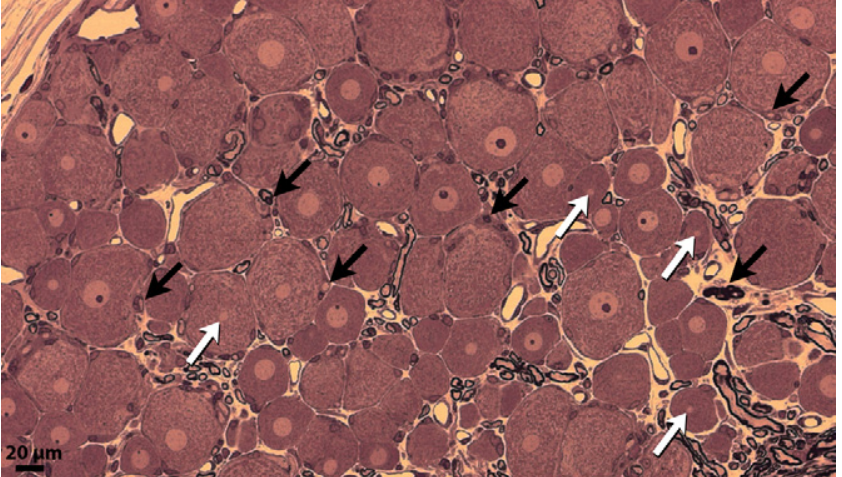

Fig. 2. Micrograph of a histological section taken from $T_{3}$-treated dorsal root ganglion. The sensory neurons can easily be distinguished from satellite and Schwann cells (black arrows) by their pale round nuclei with one or two dark nucleoli. Even when the nuclei are cut at their ends they remain identifiable (white arrows).

the basis of their large size and large nuclei bounded by a defined nuclear membrane, staining more lightly than the surrounding cytoplasm and containing at least one or more obvious nucleolar profiles which are darkly stained (Figs. 1 and 2).

The determination of the number of neurons in PBS-control and in $T_{3}$-treated stacks was based on the identification of the nuclei; each nucleus was identified, marked once at the level of the nucleolus and followed in the consecutive images of sections. On control or $\mathrm{T}_{3}$-treated DRG sections, the diameter of the nucleus of sensory neurons varied from 4 to $24 \mu \mathrm{m}$. The mean separating distance between the nuclei was around $24 \mu \mathrm{m}$ and varied from 12 to $50 \mu \mathrm{m}$. Even when the nuclei are cut at their ends they remain identifiable without any difficulty (Fig. 2). All the nuclei identified in the PBS or in $T_{3}$ DRG stacks were recorded. The total number of sensory neurons in each DRG stack corresponds to the total number of recorded nuclei. Each nucleus was visualized as a sphere using the Solid Views program (Microbrightfield Inc.). The lateral and top views of the total labelled nuclei showed a higher density in the $\mathrm{T}_{3}$-treated stack (2057 nuclei) than in the PBS-control (1096 nuclei) stack (Fig. 3).

\subsection{The reliability and validity of physical disector results based on direct counting}

In a first step we used the images of the reconstructed sections to study the reliability of the disector method (see Section 2). The position within the ganglion of the horizontal sections constituting the first pair was selected randomly between the first 10th sections. To investigate the role of a random start on the evaluation of the neuron numbers, we estimated the number of sensory neurons beginning either with the 3rd, 5 th or 7 th section in the first 10 sections from each DRG stack. The results of the three estimations revealed that the choice of the first section does not change the estimated number of neurons. In fact, the number of estimated neurons corresponding to each disector pair is tightly similar (Fig. 4) in the three experimental counts either in PBS-control (Pearson $R=0.98$; $P<0.001$ ) or in $T_{3}$-treated (Pearson $R=0.97 ; P<0.001$ ) stacks.

We also studied the role of distance on the number of estimated neurons by using increasing distances between the consecutive disector pairs $(0,10,20,30,40$ or $50 \mu \mathrm{m})$ and reducing the number of analysed pairs. The comparison between the number of neurons determined by direct counting and the number of neurons estimated by physical disector showed clearly that, in these conditions, the size of error does not exceed 6\% (Fig. 5). Therefore, $50 \mu \mathrm{m}$ is an acceptable distance between two consecutive pairs.
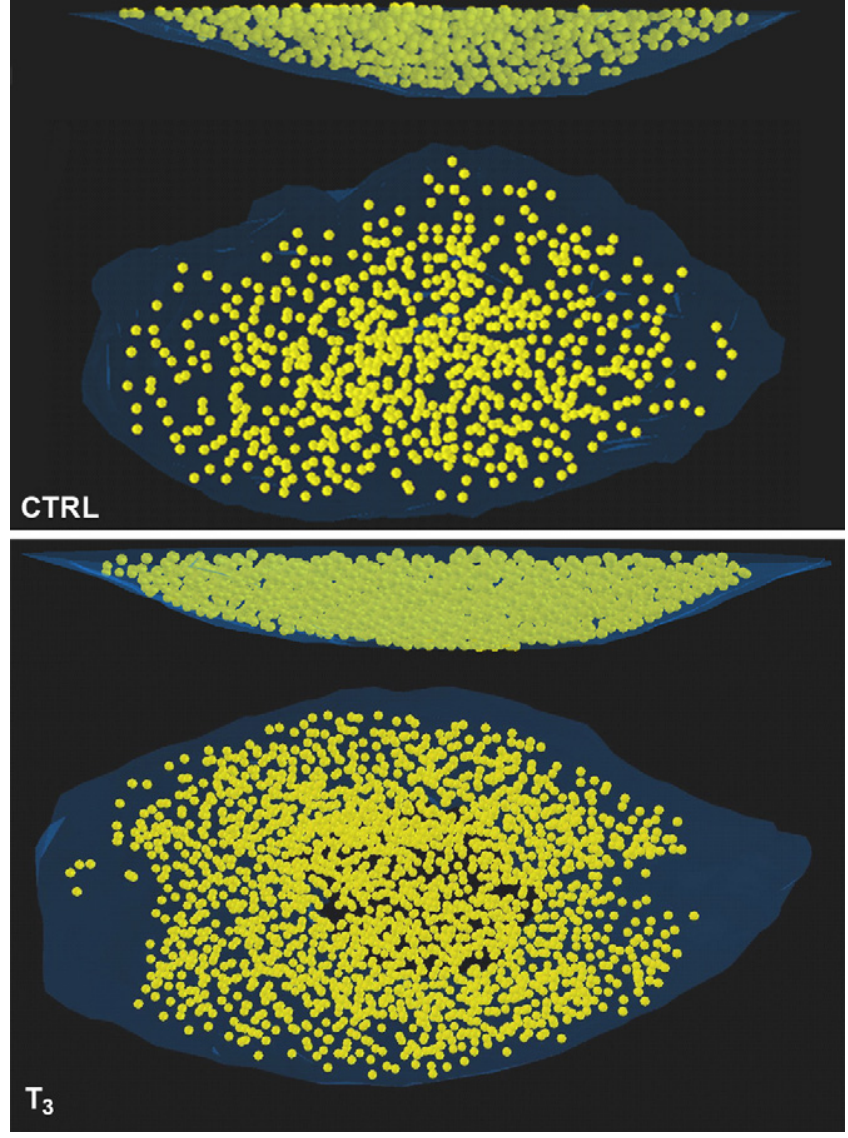

Fig. 3. Lateral and top views of the total number of neuronal nuclei in 200 consecutive DRG sections taken form PBS-control (1096 nuclei) and $\mathrm{T}_{3}$-treated (2057 nuclei) ganglia. Each nucleus is visualized as a sphere, using the Solid Views program (Microbrightfield Inc.). Note the higher density of nuclei in $\mathrm{T}_{3}$-treated DRG stack compared with PBS-control stack.

\subsection{Estimation of the number of neurons by simulation using a Matlab program and computer ganglion model}

To study the influence of a large separating distance between the consecutive disector pairs on the estimated neuron number, we created a computer model of the ganglion based on the coordinates of nucleoli recorded in the direct counting of control and $\mathrm{T}_{3}$-treated stacks. On this ganglion model we simulated 130 disector pairs perpendicularly to the long axis of the ganglia. Increasing the distances between the two consecutive pairs from 0 to $200 \mu \mathrm{m}$, reduced the number of analysed pairs from 130 to 6 pairs. The comparison between the true number of neurons obtained by direct counting and the number of neurons estimated by physical disector in our simulation conditions confirmed that when the distance is relatively small $(<60 \mu \mathrm{m})$, the variation and the size of error is acceptable and does not exceed $6 \%$. In contrast when the distance between two pairs is larger than $60 \mu \mathrm{m}(70-200 \mu \mathrm{m})$ the size of error increases rapidly to $27 \%$ (Fig. 6). Moreover, when the separating distances between two consecutive dissector pairs increase from 0 to $100 \mu \mathrm{m}$, the total number of the tops (the nuclei present only in the top section of the dissector pair) decreases in control PBS from 1096 to 99 and in $T_{3}$-treated DRG stacks from 2057 to 216 (Fig. 7). Therefore, the results of simulations on the ganglion model demonstrate that the reliability of the physical disector method is tightly related to the separating distance between the two consecutive pairs and to the number of analysed disector pairs. 

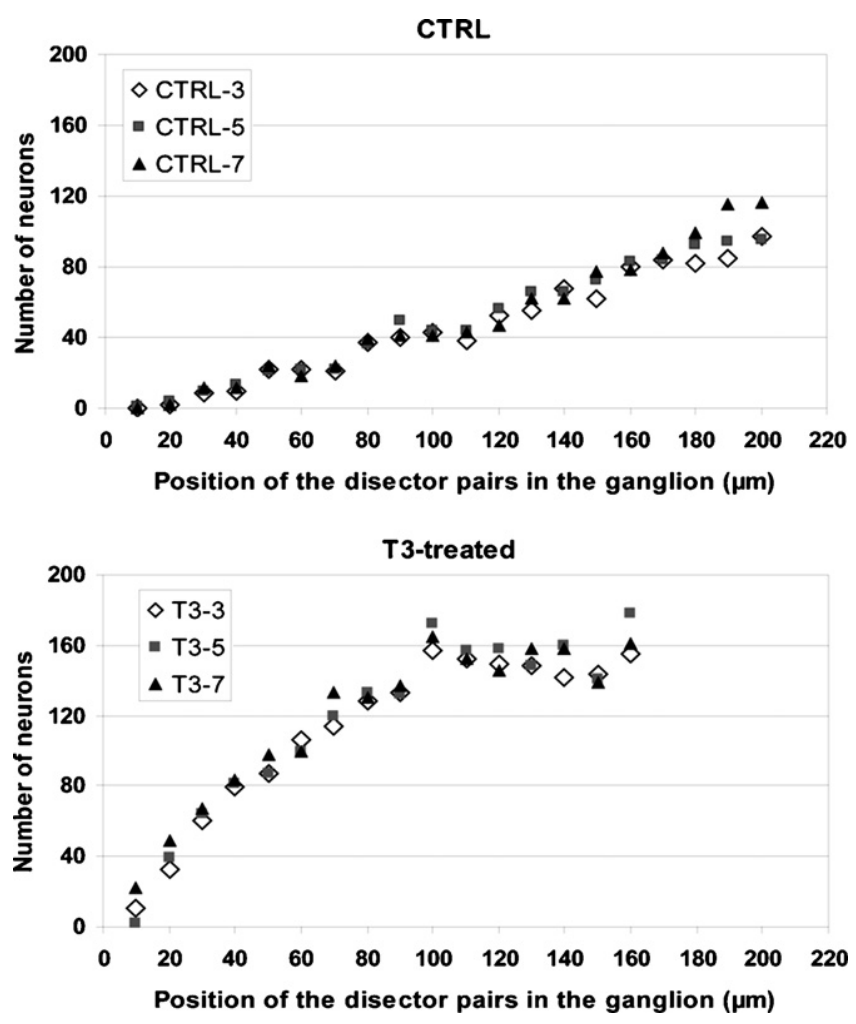

Fig. 4. Three different estimations of the number of neurons in control and $T_{3}-$ treated DRG stacks beginning either with the 3rd, 5th or 7th section as the first section of the first disector pair. The three markers indicate the number of estimated neurons corresponding to each disector pair. Our results show that the numbers of estimated neurons corresponding to each disector pair (taken at regular interval through the ganglion) are highly correlated $(R=0.98, P<0.001)$ in the three experimental conditions. Thus the random choice of the first section had no significant effect on the estimated number of neurons.

\section{Discussion}

The result of the present study reveals that the physical disector method provides a valid and reliable estimate if the separating distance between two consecutive pairs is not larger than $60 \mu \mathrm{m}$ in DRG from the rat. This result is based on:

(1) the reconstruction of a voxel-based, three-dimensional model from 200 semi-thin consecutive DRG sections (a volumetric dataset).

(2) estimation and determination of the number of sensory neurons in each stack respectively by physical disector and direct counting methods.

(3) simulation of 130 vertical disector pairs on a computer ganglion model, to study the effect of large distance separating the consecutive disector pairs on the number of estimated neurons.

\subsection{Neuron counts and stereological techniques}

The counts of nerve cells, in sections prepared for light microscopy, allow the comparison of experimental and control conditions, and help to assess the loss of neurons following nerve lesion or neuropathy. If the counting is based on reliable methods, it provides crucial information; if not, it can be seriously misleading. The use of inappropriate stereological methods usually results in a high variation in the neurons counts (Guillery and August, 2002; Schmalbruch, 1987). At the present time, no method of counting can be certified as free from error, however, the emergence of a new generation of stereological techniques has provided a more pre-
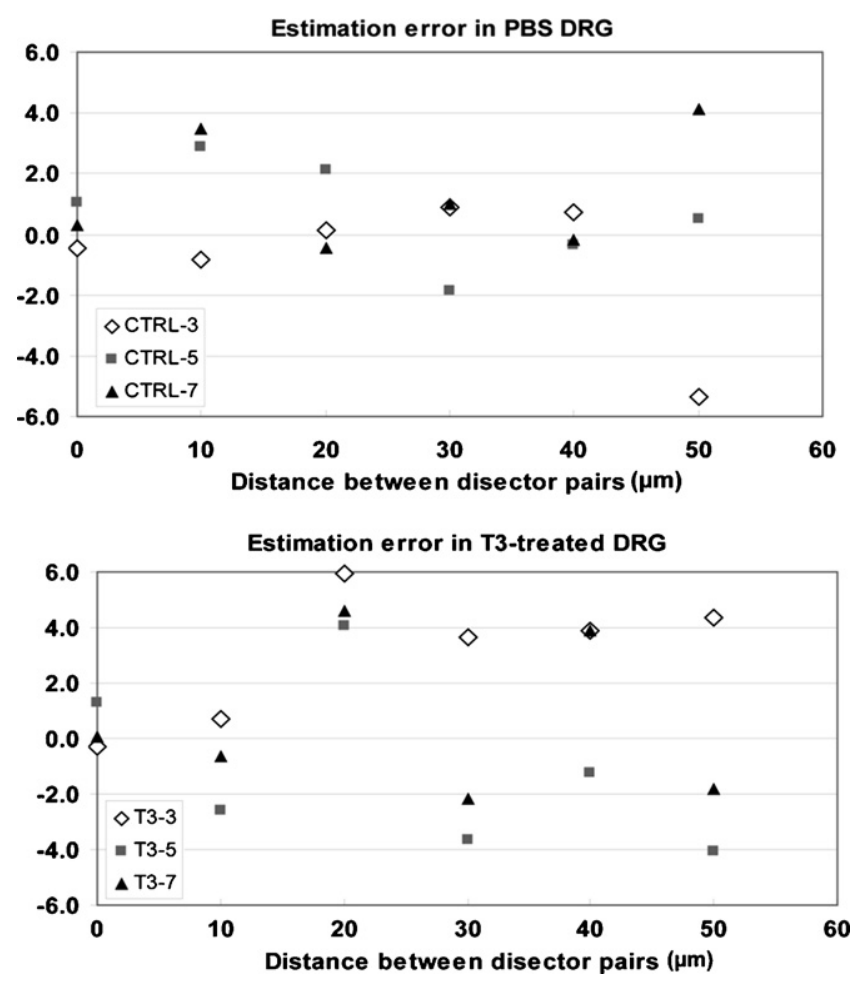

Fig. 5. Size of error between the results of physical disector and direct counting. The comparison between the true number of neurons determined by direct counting and the number of neurons estimated by physical disector using various distances between the consecutive pairs shows that the size of error does not exceed $6 \%$ when the distances vary from 0 to $50 \mu \mathrm{m}$.

cise estimation of the number of neurons when they are correctly applied.

\subsection{The importance of the choice of methods and other parameters to estimate the number of neurons}

One of the most important tasks in all quantitative approaches is choosing the method to determine the number of cells. Sources of systematic bias in morphometric data arise from faulty models or methods, assumptions and correction factors (Mouton, 2002). The most straightforward, accurate, and reliable means to determine neuron number is to follow each neuron through consecutive sections (Saper, 1997; von Bartheld, 2001). Even under the most favourable circumstances, this method is tedious and therefore it is inconceivable to use it in all situations.

The physical disector proposed in 1984 by Sterio (1984), Braendgaard and Gundersen (1986), Coggeshall (1992) has been shown to be useful to estimate the neuron number from histological sections (Gundersen et al., 1988; Saper, 1996; Coggeshall and Lekan, 1996). This method is based on sampling sections called disector pairs separated by a known distance (Tandrup, 1993, 2004; West, 2002). Since the physical disector method is a design-based sampling, several factors play a critical role in the reliability and validity of the results. The aspect of the organ which is used, the ability to identify the cells, the even or uneven distribution of the cells inside the organ, the number of disector pairs analysed, are all factors which may play an important role in the reliability of the results.

To have a good estimations using the physical disector method, the distance between the two sections from the same pair, should be proportionally linked to the diameter of the counted cells. The recommended protocol uses a separating distance roughly equiv- 
(A)
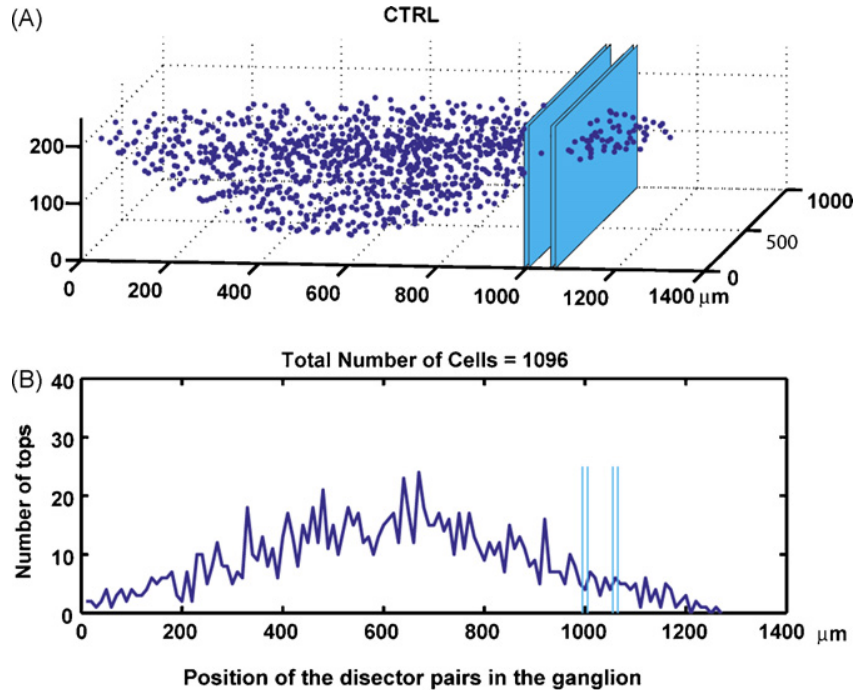

Percentage of error

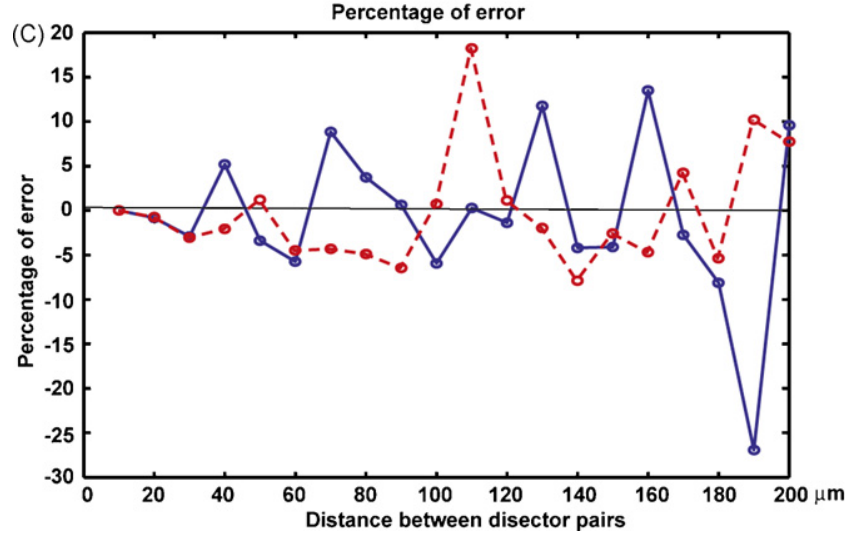

Fig. 6. Computer simulation of 130 vertical disector pairs taken perpendicularly to the long axis of the ganglion model. (A) 3D-view of the PBS-control ganglion model based on direct counting results with two simulated disector pairs. Our computer model uses the three-dimensional coordinates centers of the nuclei. The nuclei were simulated as spheres of $20 \mu \mathrm{m}$ diameter. The computer ganglion model is displayed in a rectangular parallelepiped of $200 \mu \mathrm{m}$ depth, $1300 \mu \mathrm{m}$ length and $800 \mu \mathrm{m}$ width. One dissector pair is defined by two $1 \mu \mathrm{m}$ thick sections separated by $8 \mu \mathrm{m}$. The separating distance between the two example dissector pairs is around $50 \mu \mathrm{m}$. (B) Diagram illustrating the number and the distribution of "tops" at each level of simulated physical disector pair on PBS-control ganglion. (C) Size and variation of error between the results of direct counting and the results of physical disector on the simulated models of ganglia using an increasing separation distance between the two consecutive pairs (from 0 to $200 \mu \mathrm{m}$ ). Our results show that the large distance between two consecutive pairs increases the variation and the size of error. Solid line: PBS-control, dashed line: $\mathrm{T}_{3}$-treated DRG.

alent to half the diameter of nucleus. If the separating distance between the two sections is larger than this, the risk is that small cells hidden between the two sections, will not be counted. In contrast, if the distance is very small, only few tops may be counted (Coggeshall and Lekan, 1996; West, 2002).

The fact that sensory neurons in DRG can be easily identified facilitates the counting of neuron number (La Forte et al., 1991; Tandrup, 2004). Another advantage of DRG is that we can precisely match the two sections from the same disector pair by using the edge of the DRG section in addition to other landmarks such as the nuclear profiles and blood vessels etc.

However, the uneven distribution of sensory neurons inside the ganglion is well described, which is due to the presence of bundles of fibers intermingled with the neuron cell bodies. In addition, the histological sections taken from the middle of the ganglion contain a high number of neurons while the sections taken from

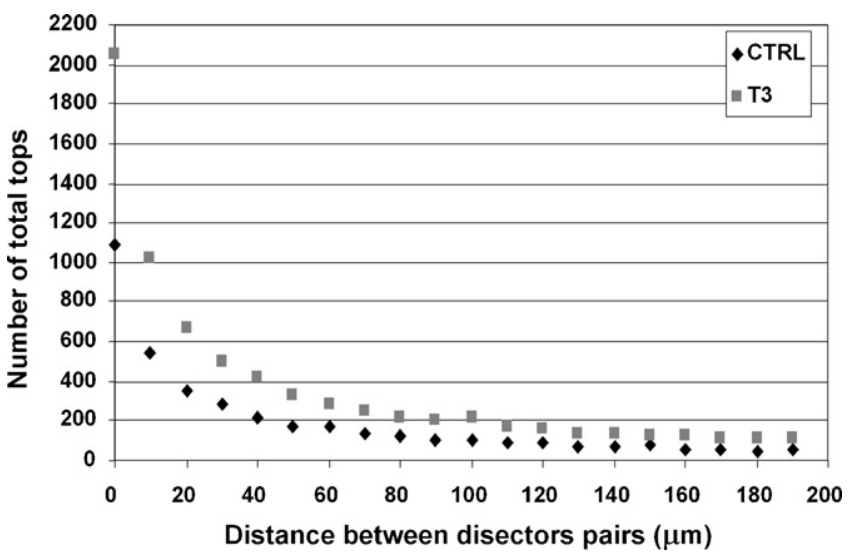

Fig. 7. Relation between the total number of "tops" and the increasing separating distance between the two consecutive disector pairs, derived from the computer ganglion model of PBS-control and $\mathrm{T}_{3}$-treated $\mathrm{DRG}$.

the two ends of the ganglion contain a small number of neurons (Coggeshall et al., 1994; Schenker et al., 2003; Tandrup, 2004). To give an equal opportunity of all the DRG regions to be sampled, the systematic random sampling must be applied (West, 1993, 2002; Geuna, 2000). In our present study we made three independent estimates by taking the 3rd, 5th or 7th section as the first section constituting the first pair. Our results revealed that the number of estimated neurons is tightly similar in the three experimental counts either in PBS-control or in $\mathrm{T}_{3}$-treated DRG section stacks (Fig. 5). Therefore, our data confirmed that systematic random sampling gives equal probability to the different part of the ganglion to be sampled, and consequently produces reliable results.

Logically to reduce the impact of an uneven distribution of neurons within the ganglion on the estimated number of neurons, the number of analysed disector pairs must be increased and the separating distance between two consecutive pairs reduced. In this regard, several investigators had reported that the reliability of the physical disector method is increased when the number of disector pairs is increased, especially when the distribution of neurons is not uniform in the organ. Sampling only a few small regions within the ganglion could lead to substantial error of estimates (Popken and Farel, 1996, 1997; Farel, 2002). However, because the analysis of a large number of disector pairs is time consuming, some investigators examine only a small number of samples, and on the basis of the data obtained, they make statements about the total cell number. This process of extension from a small number of samples to the general number can induce a large error (Popken and Farel, 1996). However, to our knowledge no study was made to determine the separating distance between the disector pairs, which allows the physical disector method to give precise results in the rat DRG. In this study, the use of two approaches (direct counting and physical dissector) revealed that $50 \mu \mathrm{m}$ is an acceptable separating distance between two consecutive pairs to allow the physical disector method to provide a valid result. In fact, the comparison between the neuron number estimated by physical disector and the true number of neurons determined by the direct counting method on the same DRG stack clearly showed that if the separating distances between the two consecutive disector pairs are $50 \mu \mathrm{m}$ or smaller $(0-50 \mu \mathrm{m})$, the size of error between the results of the two methods does not exceed 6\%, which seems a reasonable bias (von Bartheld, 2002).

Furthermore, the devised computer model based on the coordinates of the nuclei recorded in the direct count and the simulation of 130 disector pairs perpendicular to the long axis of the dorsal root allowed to test the effect of increasing distance $(0-200 \mu \mathrm{m})$ 
between the disector pairs on the estimated number of neurons. Here again the results of this approach demonstrated that when the two consecutive disector pairs are separated by $50 \mu \mathrm{m}$, the variation and the size of error remained small (less than 6\%). In contrast, when the separating distance between consecutive pairs is larger than $60 \mu \mathrm{m}(70-200 \mu \mathrm{m})$, the variation and the size of error increased rapidly (27\%) even when the number of analysed disector pairs is higher than 6 (18-6 pairs). The rationale for using the computer model of a part of the ganglion comes from the fact that the real complete serial reconstruction requires intensive labour and usually cannot be completed in a reasonable time. Thus the computer ganglion model allows to test the effect of some parameters on the estimated cell number and to confirm/or disprove the results of other methods without consuming much of time.

Therefore, on the basis of our data we conclude that to obtain accurate and reliable results by using the physical disector method we must analyse a large number of disector pairs separated by a distance which should not exceed $60 \mu \mathrm{m}$. These recommendations are also valid when the cross semi-thin sections are cut perpendicularly to the long axis of the dorsal root. In fact, the estimation of neuron number by the physical disector method in a ganglion sectioned perpendicularly to the long axis and using $50 \mu \mathrm{m}$ as a separating distance between the consecutive pairs provided an estimated total neuron number similar to the mean number of neurons which we obtained in our previous work (Schenker et al., 2003). However, the disadvantage of using cross-sections is to cut a large number of sections which increases the histological workload several folds. Furthermore, in the latter case the obligation arises to analyse a large number of disector pairs (48 pairs) compared to 20 disector pairs when the DRG is sectioned parallel to the long axis. These remarks agree with observations reported by Tandrup (2004).

\subsection{Physical disector and direct counting methods confirmed that $T_{3}$-treatment reduced the loss of axotomized sensory neurons}

Since our interest is focused on the study of the effect of a local administration of $\mathrm{T}_{3}$ on the regeneration of transected adult rat sciatic nerve (Voinesco et al., 1998; Barakat-Walter, 1999; Schenker et al., 2002), we used the physical disector method to estimate the total number of surviving neurons in lumbar DRG in control and experimental animals. To this aim we analysed a large number of disector pairs separated by $50 \mu \mathrm{m}$ and we found that the mean number of neurons in PBS-control is $8744 \pm 748$ and the mean number of neurons in $T_{3}$-treated ganglion is $12046 \pm 930$ (Schenker et al., 2003). In this study using the same DRG sections, we found that the size of error between the results of direct counting and physical disector method does not exceed 6\% (522 or 720 respectively). This small percentage of error cannot account for the difference between the neuron number in control and $\mathrm{T}_{3}$-treated ganglia. Therefore, we can infer that $\mathrm{T}_{3}$-treatment rescues a large number of axotomized sensory neurons from death.

In conclusion, the volumetric datasets reconstructed from 200 consecutive DRG semi-thin sections (stacks) on which the number of sensory neurons was estimated and determined by the physical disector and direct counting methods allowed us to demonstrate clearly that physical disector method provides valid and reliable results when the separating distance between the consecutive pairs is no larger than $60 \mu \mathrm{m}$. Moreover, the simulation of 130 disector pairs on a computer ganglion model showed that when the separating distance between consecutive pairs is larger than $60 \mu \mathrm{m}$, the variation and the size of error increased rapidly to $27 \%$.

\section{Acknowledgements}

The authors thank Dr. M. Price for critical reading of the manuscript. We express our gratitude to Mr. E. Bernardi for his help in the reconstruction of the ganglia and in artwork. This work was supported by grants from the Swiss National Science Foundation; No. 31 - 102175/03, SUVA Foundation, and Novartis Foundation.

\section{References}

Abercrombie M. Estimation of nuclear population from microtome sections. Anat Rec 1946;94:239-47.

Arvidsson J, Ygge J, Grant G. Cell loss in lumbar dorsal root ganglia and transganglionic degeneration after sciatic nerve resection in the rat. Brain Res 1986;373:15-21.

Barakat-Walter I. Role of thyroid hormones and their receptors in peripheral nerve regeneration. J Neurobiol 1999;40:541-59.

Braendgaard $\mathrm{H}$, Gundersen HJG. The impact of recent stereological advances on quantitative studies of the nervous-system. J Neurosci Methods 1986;18:39-78.

Coggeshall RE. A consideration of neural counting methods. Trends Neurosci 1992;15:9-13.

Coggeshall RE, La Forte R, Klein CM. Calibration of methods for determining numbers of dorsal root ganglion cells. J Neurosci Methods 1990;35:187-94.

Coggeshall RE, Lekan HA. Methods for determining numbers of cells and synapses: a case for more uniform standards of review. J Comp Neurol 1996;364:6-15.

Coggeshall RE, Pover CM, Fitzgerald M. Dorsal root ganglion cell death and surviving cell numbers in relation to the development of sensory innervation in the rat hindlimb. Brain Res Dev Brain Res 1994;82:193-212.

de Groot DM, Bierman EP. A critical evaluation of methods for estimating the numerical density of synapses. J Neurosci Methods 1986;18:79-101.

Farel PB. Trust, but verify: the necessity of empirical verification in quantitative neurobiology. Anat Rec 2002;269:157-61.

Geuna S. Appreciating the difference between design-based and model-based sampling strategies in quantitative morphology of the nervous system. J Comp Neurol 2000;427:333-9.

Guillery RW, August BK. Doubt and certainty in counting. In: Sutula T, Pitkanen A, editors. Progress in brain research; 2002. p. 25-42.

Gundersen HJG, Bagger P, Bendtsen TF, Evans SM, Korbo L, Marcussen N, et al. The new stereological tools-disector, fractionator, nucleator and point sampled intercepts and their use in pathological research and diagnosis. Apmis 1988;96:857-81.

Howard CV, Reed MG. Unbiased stereology. Three-dimensional measurement in microscopy. New York: Springer; 1998. pp. 246.

Konigsmark BW. Methods of the counting of neurons. In: Nauta WJH, Ebbeson SOE, editors. Contemporary research methods in neuroanatomy. Berlin: Springer; 1970. p. 315-38.

La Forte RA, Melville S, Chung K, Coggeshall RE. Absence of neurogenesis of adult rat dorsal root ganglion cells. Somatosens Mot Res 1991;8:3-7.

Liss AG, af Ekenstam FW, Wiberg M. Cell loss in sensory ganglia after peripheral nerve injury. An anatomical tracer study using lectin-coupled horseradish peroxidase in cats. Scand J Plast Reconstr Surg Hand Surg 1994;28:177-88.

Ljungberg C, Novikov L, Kellerth JO, Ebendal T, Wiberg M. The neurotrophins NGF and NT-3 reduce sensory neuronal loss in adult rat after peripheral nerve lesion. Neurosci Lett 1999;262:29-32.

McKay HA, Brannstrom T, Wiberg M, Terenghi G. Primary sensory neurons and satellite cells after peripheral axotomy in the adult rat: timecourse of cell death and elimination. Exp Brain Res 2002;142:308-18.

Mouton PR. Principles and practices of unbiased stereology: an introduction for bioscientists. Baltimore: The Johns Hopkins University Press; 2002.

Popken GJ, Farel PB. Reliability and validity of the physical disector method for estimating neuron number. J Neurobiol 1996;31:166-74

Popken GJ, Farel PB. Sensory neuron number in neonatal and adult rats estimated by means of stereologic and profile-based methods. J Comp Neurol 1997;386:8-15.

Pover CM, Coggeshall RE. Verification of the disector method for counting neurons, with comments on the empirical method. Anat Rec 1991;231:573-8.

Pu SF, Zhuang HX, Marsh DJ, Ishii DN. Insulin-like growth factor-II increases and IGF is required for postnatal rat spinal motoneuron survival following sciatic nerve axotomy. J Neurosci Res 1999;55:9-16.

Saper CB. Any way you cut it: a new journal policy for the use of unbiased counting methods. J Comp Neurol 1996;364:5.

Saper CB. Counting on our reviewers to set the standards. J Comp Neurol 1997;386:1.

Schenker M, Kraftsik R, Glauser L, Kuntzer T, Bogousslavsky J, Barakat-Walter L. Thyroid hormone reduces the loss of axotomized sensory neurons in dorsal root ganglia after sciatic nerve transection in adult rat. Exp Neurol 2003;184:225-36.

Schenker M, Riederer BM, Kuntzer T, Barakat-Walter I. Thyroid hormones stimulate expression and modification of cytoskeletal protein during rat sciatic nerve regeneration. Brain Res 2002;957:259-70.

Schmalbruch $\mathrm{H}$. Loss of sensory neurons after sciatic nerve section in the rat. Anat Rec 1987;219:323-9.

Schmitz C, Hof PR. Design-based stereology in neuroscience. Neuroscience 2005;130:813-31. 
Sterio DC. The unbiased estimation of number and sizes of arbitrary particles using the disector. J Microsc 1984;134(Pt 2):127-36.

Tandrup T. A method for unbiased and efficient estimation of number and mean volume of specified neuron subtypes in rat dorsal-root ganglion. J Comp Neurol 1993;329:269-76.

Tandrup T. Unbiased estimates of number and size of rat dorsal root ganglion cells in studies of structure and cell survival. J Neurocytol 2004;33:173-92.

Tessler A, Himes BT, Krieger NR, Murray M, Goldberger ME. Sciatic-nerve transection produces death of dorsal-root ganglion-cells and reversible loss of substance-P in spinal-cord. Brain Res 1985;332:209-18.

Voinesco F, Glauser L, Kraftsik R, Barakat-Walter I. Local administration of thyroid hormones in silicone chamber increases regeneration of rat transected sciatic nerve. Exp Neurol 1998;150:69-81. von Bartheld CS. Comparison of 2-D and 3-D counting: the need for calibration and common sense. Trends Neurosci 2001;24:504-6.

von Bartheld CS. Counting particles in tissue sections: choices of methods and importance of calibration to minimize biases. Histol Histopathol 2002;17:639-48.

West MJ. New stereological methods for counting neurons. Neurobiol Aging 1993;14:275-85.

West MJ. Design-based counting techniques: the real problems-Reply. Trends Neurosci 1999a;22:345-6.

West MJ. Stereological methods for estimating the total number of neurons and synapses: issues of precision and bias. Trends Neurosci 1999b;22:51-61.

West MJ. Design-based stereological methods for counting neurons. Prog Brain Res 2002;135:43-51. 